\title{
HIV Drug Resistance at Patients on HAART and Transmitted HIV Drug Resistance (tHIVDR) in Treatment Naive Patients in Belarus
}

\section{Vladimir Eremin ${ }^{1 *}$, Elena Gasich ${ }^{1}$, Sviataslau Sasinovich ${ }^{1}$, Oleg Suetnov ${ }^{2}$ and Igor Kucherov ${ }^{1}$}

${ }^{1}$ Republican Research \& Practical Centre for Epidemiology \& Microbiology, Minsk, Belarus

${ }^{2}$ Gomel Regional Centre of Epidemiology \& Public Health, Gomel, Belarus

\begin{abstract}
Of the 59 patients (adults) who have been identified to have a virus with a high level resistance to HAART drugs, in 38 (64.4\%) patients mutation M184V/I was identified. In 27 (45.8\%) patients (13 female and 14 male) K103N mutation was detected. In $20(33.9 \%)$ patients (16 male and 4 female) we found G190G/S/A mutation. Of the 18 childrenpatients, born to HIV-infected mothers, in $15(83.3 \%)$ cases (9 girls and 6 boys) we detected HIV resistance mutation M184V. In $10(55.6 \%)$ and $2(11.1 \%)$ cases mutations in position G190S and K103S were found, respectively. Of 82 samples collected from newly diagnosed HIV-infected antiretroviral naïve patients only 6 samples (7.3\%) had other resistance mutations which can be classified as 'minor' or 'other' according to HIVDR database of Stanford University: L10V - PI minor mutation associated with resistance to most PIs when present with other mutations; L33F - PI minor mutation selected by FPV/r, DRV/r, LPV/r, ATV/r, and TPV/r, and contributes decreased susceptibility to these PIs; V118I - accessory mutation usually occurred with multiple TAMs and contributes some resistance to each of the NRTIs including 3TC and FTC; T74S is 'other' mutation associated with reduced NFV susceptibility; and V108I - accessory mutation, causes low-level resistance to NVP and EFV.
\end{abstract}

Keywords: HIV; IDUs; Resistance; Mutations; Sequencing; Subtypes

\section{Introduction}

One of the main factors influencing the level of efficiency of highly active antiretroviral therapy (HAART) is the high degree of variability of certain regions of the genome of human immunodeficiency virus (HIV) that leads to the emergence of mutations associated with resistance to antiretroviral therapy (ART). ART treatment regimen use more than 30 drugs of 5 different classes, but, unfortunately, we already identified some mutations in the genome of the virus which cause developing HIV resistance to these drugs [1,2]. At present day there is no alternative to HAART, although it cannot be considered $100 \%$ effective treatment, but HAART regimen help and significantly improve the living status of HIV-infected patients. Early diagnoses of HIV resistance mutations development and consequent changing of treatment regime are the bases of HAART efficiency.

Belarus witnesses consistently high growth of HIV/AIDS cases. Over the last 5 years more than 1,000 new HIV-infection cases were revealed annually. By August $1^{\text {st }} 2013$ - 15,038 (158 infected people per 100 thousand population) patients with HIV/AIDS were registered, during 7 months period in the year 2013 - 860 new HIV infection cases were officially registered (Figure 1). The largest number of HIV/AIDS cases were reported in the age group from 15 to 29 years (8613 cases), but at present day the majority of new HIV cases are being recorded in the age group from 30 up to 45 years, the primary mechanism of HIV transmission is sexual. For instance, if in year 2012 the proportion of sexual HIV transmission mechanism amounted to $77.2 \%$ of all new cases; in 2013 already $83 \%$ of the patients were infected through sexual rout.

Currently, in Belarus - 4,500 patients are on HAART, while in year 2008 only 1,200 patients were receiving this therapy, in year 2004 - only 15 patients. It is planned, that by the year 2015 - 7,000 patients will be on ART. In this report we present the results of studies on detecting the resistance mutations in HIV/AIDS patients on HAART as well as at HIV/AIDS patients which are not receiving antiretroviral therapy in Belarus.

\section{Materials and Methods}

The article presents data on the identification of high-level resistance mutations developed by 77 patients (59 adults and 18 children) who have been on HAART during the period from year 2008 to year 2013. Information on these patients is shown in Tables 1 (adult) and 2 (children).Blood plasma from 96 patients initially identified with HIV/AIDS, who were not receiving antiretroviral therapy, has been investigated for the presence of primary resistance mutations.

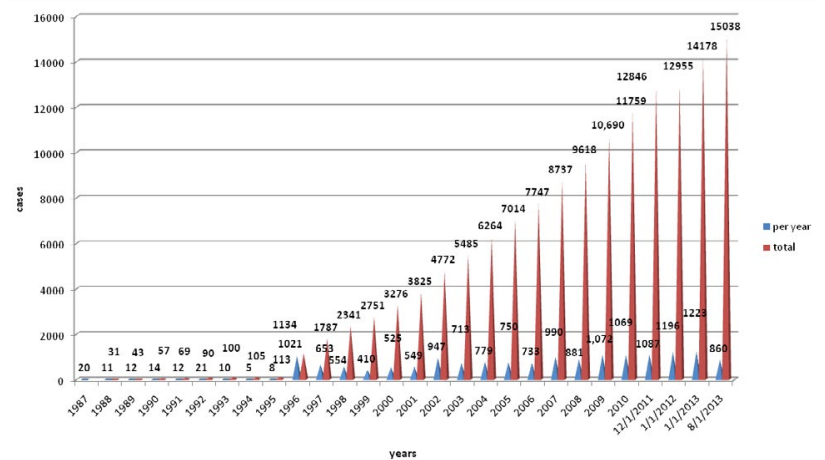

Figure 1: HIVIAIDS dynamics in Belarus (1987 - 01.08.2013)

*Corresponding author: Vladimir Eremin, Republican Research \& Practical Centre for Epidemiology \& Microbiology, Minsk, Belarus, E-mail: veremin@mail.ru

Received May 27, 2013; Accepted October 29, 2013; Published November 01, 2013

Citation: Eremin V, Gasich E, Sasinovich S, Suetnov O Kucherov I (2013) HIV Drug Resistance at Patients on HAART and Transmitted HIV Drug Resistance (tHIVDR) in Treatment Naive Patients in Belarus. J AIDS Clin Res 4: 258. doi: 10.4172/2155-6113.1000258

Copyright: (C) 2013 Eremin V, et al. This is an open-access article distributed under the terms of the Creative Commons Attribution License, which permits unrestricted use, distribution, and reproduction in any medium, provided the original author and source are credited. 
Citation: Eremin V, Gasich E, Sasinovich S, Suetnov O Kucherov I (2013) HIV Drug Resistance at Patients on HAART and Transmitted HIV Drug Resistance (tHIVDR) in Treatment Naive Patients in Belarus. J AIDS Clin Res 4: 258. doi: 10.4172/2155-6113.1000258

Page 2 of 9

\begin{tabular}{|c|c|c|c|c|c|c|c|c|c|c|}
\hline NN & $\begin{array}{l}\text { GenBank data, } \\
\text { ID,region }\end{array}$ & $\operatorname{sex}$ & age & $\begin{array}{l}\text { HIV } \\
\text { infection/ } \\
\text { HAART } \\
\text { started }\end{array}$ & $\begin{array}{l}\text { Rout of } \\
\text { infection }\end{array}$ & $\begin{array}{l}\text { HIV } \\
\text { subtype }\end{array}$ & $\begin{array}{l}\text { Data of } \\
\text { high/level } \\
\text { mutations } \\
\text { detection }\end{array}$ & Pls & NRTIs & NNRTIs \\
\hline 1 & 2 & 3 & 4 & & & 5 & & 6 & 7 & 8 \\
\hline 1. & Vit-2-A HE657489, & f & 1978 & $2002 / 2005$ & heterosex & A1 & 2008 & - & $\begin{array}{l}\text { D67N; K70R; M184V; } \\
\text { K219Q }\end{array}$ & V179DV; Y188L \\
\hline 2. & Gom-28-A - & $\mathrm{m}$ & 1970 & $1998 / 2004$ & IDU & A1 & 2008 & $\begin{array}{l}\text { D30N; } \\
\text { N88D;+ } \\
\text { минорные: } \\
\text { L10V; T74S }\end{array}$ & $\begin{array}{l}\text { M41L; D67N; K70R; T215F; } \\
\text { K219Q }\end{array}$ & G190S \\
\hline 3. & $\begin{array}{l}\text { FR686468,MnObl- } \\
\text { 28-cpx; }\end{array}$ & f & 1982 & $2008 / 2008$ & heterosex & CRF06_cpx & 2010 & - & - & K103N; K238T \\
\hline 4. & $\begin{array}{l}\text { HE657525 } \\
\text { MnObl-24-A }\end{array}$ & $\mathrm{m}$ & 1968 & $2008 / 2009$ & heterosex & A1 & 2010 & - & M184V & $\begin{array}{l}\text { K103N; V108IV; } \\
\text { P225HP }\end{array}$ \\
\hline 5. & $\begin{array}{l}\text { Br-18-A; } \\
\text { HE657536 }\end{array}$ & $\mathrm{m}$ & 1974 & $2006 / 2009$ & heterosex & A1 & 2010 & - & M184V & K101E; E138A; G190A \\
\hline 6. & $\begin{array}{l}\text { MnObl-29-A } \\
\text { HE657524 }\end{array}$ & f & 1969 & $2007 / 2009$ & heterosex & A1 & 2010 & - & - & G190S \\
\hline 7. & $\begin{array}{l}\text { MnObl-25-A; -; } \\
\text { HE657522ИHН, }\end{array}$ & f & 1982 & $2003 / 2008$ & IDU & A1 & $2010 / 2012$ & - & V181I; M184V & K103N \\
\hline 8. & $\begin{array}{l}\text { MnObl-27-cpx; C } \\
\text { FR686469 }\end{array}$ & $\mathrm{m}$ & 1980 & $2008 / 2008$ & heterosex & CRF06_cpx & 2010 & - & M184V & K103N \\
\hline 9. & $\begin{array}{l}\text { MnObl-30- } \\
\text { A1HE657516 }\end{array}$ & $\mathrm{m}$ & 1979 & $1998 / 2006$ & IDU & A1 & 2010 & - & - & K103N; E138A \\
\hline 10. & $\begin{array}{l}\text { MnObl-35- } \\
\text { AHE657541 }\end{array}$ & $\mathrm{m}$ & 1981 & $2007 / 2009$ & heterosex & A1 & 2010 & - & - & K103N; E138A \\
\hline 11. & $\begin{array}{l}\text { MnObl-31- } \\
\text { AHE657526 }\end{array}$ & $f$ & 1985 & $2003 / 2008$ & heterosex & A1 & 2010 & - & V118I; M184V & K103N; P225H \\
\hline 12. & $\begin{array}{l}\text { MnObl-34- } \\
\text { A;HE657540 }\end{array}$ & $\mathrm{m}$ & 1976 & $2002 / 2006$ & IDU & A1 & 2010 & - & $\begin{array}{l}\text { Y115F; V118I; M184V; } \\
\text { T215F }\end{array}$ & E138A \\
\hline 13. & $\begin{array}{l}\text { MnObl-01-A-BY; } \\
\text { HE577613.1 }\end{array}$ & $\mathrm{m}$ & 1959 & $2008 / 2008$ & heterosex & A1 & 2010 & - & M184V & E138A \\
\hline 14. & $\begin{array}{l}\text { MnObl-16-A, } \\
\text { HE657523 }\end{array}$ & $f$ & 1978 & $2003 / 2006$ & IDU & $\mathrm{A} 1$ & 2008 & - & - & K103N \\
\hline 15. & $\begin{array}{l}\text { MnObl-17- } \\
\text { A;HE657529 }\end{array}$ & $f$ & 1987 & $2004 / 2008$ & IDU & A1 & 2010 & L10I & V118I, M184V, T215F & K101H; G190S \\
\hline 16. & $\begin{array}{l}\text { MnObl-06-A; } \\
\text { HE577618, }\end{array}$ & $\mathrm{m}$ & 1975 & $1996 / 2007$ & IDU & A1 & 2010 & - & K65R, M184V & K103N; P225H \\
\hline 17. & $\begin{array}{l}\text { MnObl-04-A; } \\
\text { HE577616 }\end{array}$ & $\mathrm{m}$ & 1971 & $2002 / 2007$ & IDU & A1 & 2010 & L10IL & V118I & K103N \\
\hline 18. & Vit-3-A; HE657491 & $\mathrm{m}$ & 1981 & $2001 / 2009$ & IDU & A1 & 2011 & - & T69NT, M184V & Y181CFGV \\
\hline 19. & Gom-37A1 & $\mathrm{m}$ & 1977 & $1998 / 2006$ & IDU & A1 & 2008 & - & T215ST & G190GS \\
\hline 20. & $\begin{array}{l}\text { MnObl- } \\
\text { 40AHF679227.1 }\end{array}$ & $f$ & 1974 & $2007 / 2010$ & heterosex & A1 & 2011 & - & M184V & K103KN \\
\hline 21. & MnObl-41 & $\mathrm{m}$ & 1979 & $2002 / 2009$ & IDU & A1 & 2011 & - & M184V & K103N; P225H \\
\hline 22. & $\begin{array}{l}\text { MnObl-42Рудик } \\
\text { HF679229.1 }\end{array}$ & $f$ & 1985 & $2007 / 2009$ & heterosex & A1 & 2011 & - & - & K103N \\
\hline 23. & $\begin{array}{l}\text { Br-21- } \\
\text { A;HF679265.1 }\end{array}$ & $\mathrm{m}$ & 1972 & $1998 / 2008$ & IDU & A1 & 2011 & & A62V, T69S, L74LV, M184I & V90I, K101E, G190S \\
\hline 24. & $\begin{array}{l}\text { Br-20- } \\
\text { AHF679264.1 }\end{array}$ & $\mathrm{m}$ & 1972 & $1998 / 2008$ & IDU & A1 & 2011 & - & D67G, K70EK, M184V & K101Q, Y181CY, G190S \\
\hline 25. & $\begin{array}{l}\text { Vit-5- } \\
\text { A;HF679261.1 }\end{array}$ & $\mathrm{m}$ & 1976 & $2001 / 2010$ & IDU & A1 & 2011 & - & L74V, Y115F, M184V & V90I, G190S, H221Y \\
\hline 26. & $\begin{array}{l}\text { Br-22-A; } \\
\text { HF679266.1 }\end{array}$ & $f$ & 1975 & $2003 / 2010$ & IDU & A1 & 2012 & - & M184V & K103N \\
\hline 27. & $\begin{array}{l}\text { Mn-29- } \\
\text { A;HF679247.1 }\end{array}$ & $\mathrm{m}$ & 1976 & $2002 / 2011$ & IDU & A1 & 2012 & - & $\begin{array}{l}\text { M41L, D67N, K70R, M184V, } \\
\text { L210W, T215Y, K219E }\end{array}$ & - \\
\hline 28. & $\begin{array}{l}\text { Mn-30-A; } \\
\text { HF679248.1 }\end{array}$ & $f$ & 1975 & $1998 / 2003$ & heterosex & A1 & 2012 & $\begin{array}{l}\text { M46I, I54V, } \\
\text { V82A }\end{array}$ & M184V & - \\
\hline 30. & $\begin{array}{l}\text { MnObl-44- } \\
\text { A;HF679219.1 }\end{array}$ & f & 1983 & $2006 / 2010$ & heterosex & A1 & 2012 & - & M184V & K103N, E138Q \\
\hline 31. & Mn-31A;HF679249 & $\mathrm{m}$ & 1983 & $2006 / 2010$ & IDU & A1 & 2012 & - & - & K103N \\
\hline 32. & Br-24-AHF679271 & $\mathrm{m}$ & 1977 & $2001 / 2009$ & IDU & A1 & 2012 & - & M184V & K103N \\
\hline
\end{tabular}




\begin{tabular}{|c|c|c|c|c|c|c|c|c|c|c|}
\hline 33. & $\begin{array}{l}\text { Br-26- } \\
\text { A;HF679270.1 }\end{array}$ & $\mathrm{m}$ & 1984 & $2002 / 2010$ & IDU & A1 & 2012 & - & - & K103N \\
\hline 34. & $\begin{array}{l}\text { Br-27- } \\
\text { A;HF679277.1 }\end{array}$ & $\mathrm{m}$ & 1955 & $1997 / 2011$ & IDU & A1 & 2012 & - & A62V, M184V & Y181C \\
\hline 35. & $\begin{array}{l}\text { Mn-33- } \\
\text { A;HF679251 }\end{array}$ & $\mathrm{m}$ & 1983 & $2004 / 2011$ & IDU & A1 & 2012 & - & A62V, K65R, M184IMV & $\begin{array}{l}\text { V90I, V179T, Y181C, } \\
\text { G190S }\end{array}$ \\
\hline 36. & Br-29-A;HF679273 & f & 1982 & $2006 / 2010$ & heterosex & A1 & 2012 & & M184V & K101E, G190A \\
\hline 37. & $\begin{array}{l}\text { MnObl-47- } \\
\text { A;HF679230 }\end{array}$ & f & 1967 & $2009 / 2009$ & heterosex & A1 & 2012 & & M184V & K103N \\
\hline 38. & $\begin{array}{l}\text { MnObl-48- } \\
\text { A;HF679235 }\end{array}$ & $\mathrm{m}$ & 1981 & $2009 / 2010$ & IDU & A1 & 2012 & & L74V, Y115F, M184V & K101E, Y181C, G190S \\
\hline 39. & $\begin{array}{l}\text { MnObl-49- } \\
\text { A;HF679232 }\end{array}$ & $\mathrm{m}$ & 1977 & $2008 / 2010$ & heterosex & A1 & 2012 & & $\begin{array}{l}\text { D67N, K70R, M184V, T215Y, } \\
\text { K219Q }\end{array}$ & K103N, P225H \\
\hline 40. & $\begin{array}{l}\text { MnObl-50-A1; } \\
\text { HF679216 }\end{array}$ & f & 1982 & $2004 / 2010$ & heterosex & A1 & 2012 & - & L74LV, M184V & K103N, Y181C \\
\hline 41. & $\begin{array}{l}\text { Gom-29- } \\
\text { A;HF679272 }\end{array}$ & $\mathrm{m}$ & 1975 & $2008 / 2009$ & heterosex & A1 & 2012 & - & $\begin{array}{l}\text { M41L, K70KN, V75IM, } \\
\text { M184V, T215Y }\end{array}$ & Y181C \\
\hline 42. & $\begin{array}{l}\text { MnObl- } \\
\text { 53A1;HF679231 }\end{array}$ & $\mathrm{m}$ & 1985 & $2006 / 2011$ & IDU & A1 & 2012 & - & - & G190GS \\
\hline 43. & MnObl-55A1 & $\mathrm{m}$ & 1984 & $2006 / 2011$ & IDU & A1 & 2012 & - & - & K103KN \\
\hline 44. & $\begin{array}{l}\text { MnObl- } \\
\text { 56A1;HF679210 }\end{array}$ & f & 1980 & $2005 / 2010$ & heterosex & A1 & 2012 & - & D67DN, M184V, T215NSTY & K101E, G190A \\
\hline 45. & $\begin{array}{l}\text { MnObl-57A1; } \\
\text { HF679212 }\end{array}$ & $\mathrm{m}$ & 1970 & $2007 / 2010$ & IDU & A1 & 2012 & - & - & K103N \\
\hline 46. & $\begin{array}{l}\text { MnObl-58A1 } \\
\text { HF679234 }\end{array}$ & $\mathrm{m}$ & 1981 & $2007 / 2010$ & IDU & A1 & 2012 & & $\begin{array}{l}\text { D67DN, K70R, M184V, } \\
\text { T215IT, K219EK }\end{array}$ & Y181CY \\
\hline 47. & MnObl-61A/B & $\mathrm{m}$ & 1975 & $1998 / 2008$ & IDU & $A / B$ & 2012 & - & - & K103S, G190A \\
\hline 48. & $\begin{array}{l}\text { MnObl- } \\
\text { 63A1,HF586690 }\end{array}$ & $\mathrm{m}$ & 1972 & $1997 / 2009$ & IDU & A1 & 2012 & - & K65KR, K70EK, M184V & K101H, Y181CY, G190S \\
\hline 49. & $\begin{array}{l}\text { Gom-30A1, } \\
\text { HF679275 }\end{array}$ & $f$ & 1989 & $2008 / 2010$ & heterosex & A1 & 2012 & - & K65R, Y115F, M184V & - \\
\hline 50. & $\begin{array}{l}\text { Mn-37B, } \\
\text { HF679255 }\end{array}$ & $\mathrm{m}$ & 1970 & $1998 / 2009$ & heterosex & B & 2012 & - & M184V & - \\
\hline 51. & $\begin{array}{l}\text { MnObl-68A1; } \\
\text { HF679223 }\end{array}$ & $\mathrm{m}$ & 1978 & $2002 / 2009$ & IDU & A1 & 2012 & - & M184V & G190S \\
\hline 52. & Mn-43A1 & $f$ & 1982 & $2004 / 2011$ & IDU & A1 & 2013 & - & - & K103KN \\
\hline 53. & MnObl-72A1, & $\mathrm{m}$ & 1973 & $1998 / 2011$ & IDU & A1 & 2013 & - & - & K103N \\
\hline 54. & MnObl-75A1 & $\mathrm{m}$ & 1978 & $2012 / 2012$ & IDU & A1 & 2013 & - & A62V, L74LV, M184I & K101E, Y181CY, G190S \\
\hline 55. & MnObl-80A1; & $\mathrm{m}$ & 1979 & $2010 / 2011$ & IDU & A1 & 2013 & - & - & K103N, Y188L \\
\hline 56. & MnObl-84A1; & $\mathrm{m}$ & 1983 & $2008 / 2010$ & IDU & A1 & 2013 & - & - & G190S \\
\hline 57. & Mn-47A1; & $\mathrm{m}$ & 1967 & $2003 / 2008$ & IDU & A1 & 2013 & - & $\begin{array}{l}\text { D67G, L74V, M184MV, } \\
\text { T215ST, K219E }\end{array}$ & K101H, Y181C, G190S \\
\hline 58. & Mn-51A1 & $\mathrm{m}$ & 1971 & $2000 / 2008$ & IDU & A1 & 2012 & & M184V & - \\
\hline 59. & MnObl-94A1 & $\mathrm{m}$ & 1974 & $2006 / 2006$ & IDU & A1 & 2013 & & $\begin{array}{l}\text { M41LM, A62V, M184V, } \\
\text { T215Y }\end{array}$ & K101E, E138A, G190S \\
\hline 60. & Gom-38A1 & $f$ & 1980 & $1999 / 2004$ & heterosex & A1 & 2008 & & - & K103N \\
\hline
\end{tabular}

Table 1: High/level resistance mutation at patients on HAART (adults).

For detecting the resistance mutations we used commercial kit «ViroSeq ${ }^{\mathrm{TM}}$ HIV-1 genotyping system v.2.0» (Abbott, USA) with an analytical sensitivity $2 \times 10^{3}$ copies $\mathrm{RNA} / \mathrm{ml}$. This kit allows sequencing of HIV-1 gene polregion encoding of proteases and $2 / 3$ of the reverse transcriptase (1800 bp). Electrophoresis of DNA fragments after sequencing PCR was performed on genetic analyzer ABI PRISM 3100-Avant (Applied Biosystems, USA).

Analysis of the obtained fragments and detecting of HIV resistance mutations to antiretroviral drugs was performed using commercial databases «ViroSeq HIV-1 genotyping system software v2.6 analysis» (Abbott, USA) and Stanford University "HIV Drug Resistance Database", and using software «Sequencing Analysis Software v.5.1.1», «BioEdit», «SeqScape v.2.6».
Phylogenetic analysis of the DNA fragments was performed with the help of the program Mega 4.1 (trees constructed by neighborjoining method).

\section{Results and Discussion}

\section{Resistance mutation in patients on HAART}

Of the 59 patients (adults) who have been identified to have a virus with a high level resistance to HAART drugs, there were 39 male (median age $37.2 \pm 4.8$ years) and 20 female (median age $33.9 \pm$ 4.92 years). 20 patients (6 male and 14 female) were infected heterohomosexually and 39 ( 33 male and 6 female) were IDUs. 35 patients came from different parts of the Minsk region, 8 patients from the city of Minsk, 8 patients from the Brest region, 5 patients from the Gomel 
Citation: Eremin V, Gasich E, Sasinovich S, Suetnov O Kucherov I (2013) HIV Drug Resistance at Patients on HAART and Transmitted HIV Drug Resistance (tHIVDR) in Treatment Naive Patients in Belarus. J AIDS Clin Res 4: 258. doi: 10.4172/2155-6113.1000258

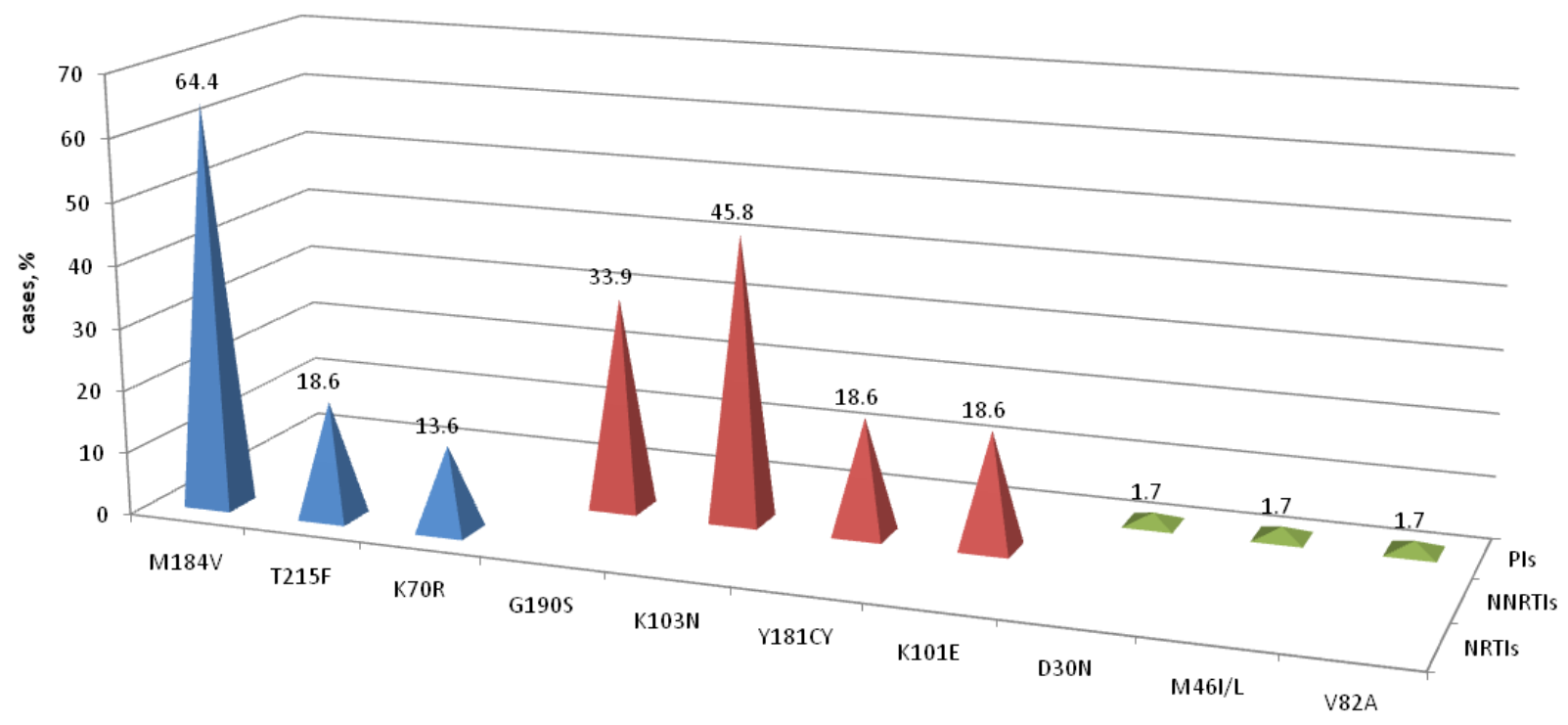

nRTIs

nNRTIs

mutations

Figure 2: Resistance mutations identified in patients on HAART (adults)

\begin{tabular}{|c|c|c|c|c|c|c|c|c|c|}
\hline № $n / n v$ & $\begin{array}{l}\text { GenBank data, } \\
\text { ID,region }\end{array}$ & sex & age & $\begin{array}{l}\text { HIV infection/HAART } \\
\text { started }\end{array}$ & HIV subtype & $\begin{array}{l}\text { Data of high/ } \\
\text { level mutations } \\
\text { detection }\end{array}$ & Pls & NRTIs & NNRTIs \\
\hline 1 & 2 & 3 & 4 & & 5 & & 6 & 7 & 8 \\
\hline 1. & $\begin{array}{l}\text { Mg-2-A/ } \\
\text { G;FN995208. }\end{array}$ & $b$ & 2003 & $2003 / 2005$ & CRF02_AG & 2009 & - & $\begin{array}{l}\text { M42L; D67N; } \\
\text { T69Xi; K70R; } \\
\text { V75M; T215F; } \\
\text { K219Q }\end{array}$ & $\begin{array}{l}\text { K101KQ; K103S; } \\
\text { G190A }\end{array}$ \\
\hline 2. & $\begin{array}{l}\text { Gom-26- } \\
\text { A;HE657433 }\end{array}$ & b & 1996 & $1996 / 2004$ & A & $2008 / 2012$ & $\begin{array}{l}\text { M46I; I54V; } \\
\text { V82F; I84V }\end{array}$ & $\begin{array}{l}\text { A62V; T69Xi; L74I; } \\
\text { Y115F; M184V; } \\
\text { L210W; T215Y }\end{array}$ & - \\
\hline 3. & $\begin{array}{l}\text { MnObl-51-A1; } \\
\text { HF679233.1 }\end{array}$ & b & 2002 & $2002 / 2006$ & A & 2008 & - & $\begin{array}{l}\text { D67N; K70R; } \\
\text { V118I; M184V; } \\
\text { T215Y; K219E }\end{array}$ & V106I; Y188L \\
\hline 4. & $\begin{array}{l}\text { Mg-10-A; } \\
\text { FR686903 }\end{array}$ & b & 2003 & $2003 / 2008$ & A & 2009 & - & $\begin{array}{l}\text { M41L; E44DE; } \\
\text { D67N; T69Xi; } \\
\text { M184V; L210W; } \\
\text { T215Y }\end{array}$ & - \\
\hline 5. & Mn-24-A,HF679242 & $\mathrm{g}$ & 2004 & $2004 / 2005$ & A & 2009 & - & $\begin{array}{l}\text { D69N; T69Xi; } \\
\text { K70R; V118I; } \\
\text { M184V; K219Q }\end{array}$ & - \\
\hline 6. & $\begin{array}{l}\text { Gom-17- } \\
\text { A;HE657430 }\end{array}$ & g & 1996 & $1996 / 2004$ & A & 2008 & $\begin{array}{l}\text { M46I; I54V; } \\
\text { V82F+ } \\
\text { L10V; T74S; } \\
\text { L89V }\end{array}$ & $\begin{array}{l}\text { L74IV, Y115F, } \\
\text { V118I, M184V, } \\
\text { T215F }\end{array}$ & $\begin{array}{l}\text { K101E, Y181CY, } \\
\text { G190S, H221Y }\end{array}$ \\
\hline 7. & $\begin{array}{l}\text { Gom-27-A; } \\
\text { HE657431 }\end{array}$ & g & 2001 & $2001 / 2008$ & A & $2010 / 2012$ & - & M184V & Y188L \\
\hline 8. & $\begin{array}{l}\text { MnObl-33-A; } \\
\text { HE657542 }\end{array}$ & g & 2008 & $2008 / 2009$ & A & 2011 & - & T69NT, M184V & Y181CFGV \\
\hline 9. & $\begin{array}{l}\text { MnObl-03- } \\
\text { A;HE577615. }\end{array}$ & g & 2004 & $2004 / 2006$ & A & 2010 & - & $\begin{array}{l}\text { D67G, M184V, } \\
\text { K219N }\end{array}$ & G190S \\
\hline 10. & Gom-32A1 & b & 2004 & $2004 / 2004$ & A & 2008 & $\begin{array}{l}\text { M46I; L90M; } \\
\text { A71T; T74S }\end{array}$ & $\begin{array}{l}\text { D67N; T69i; K70R; } \\
\text { T215FY }\end{array}$ & \\
\hline 11. & Vit-4-A; HF67926 & $b$ & 2003 & $2011 / 2011$ & A & 2012 & M46L & A62V, D67G & $\begin{array}{l}\text { V90I, K101E, } \\
\text { G190S }\end{array}$ \\
\hline 12. & $\begin{array}{l}\text { Br-23-A; } \\
\text { HF679276.1 }\end{array}$ & b & 2005 & $2005 / 2009$ & A & 2012 & - & V75MV, M184V & K101E, G190S \\
\hline 13. & $\begin{array}{l}\text { MnObl-46- } \\
\text { A;HF679224 }\end{array}$ & b & 2003 & $2003 / 2006$ & A & 2012 & - & $\begin{array}{l}\text { D67N, K70R, } \\
\text { M184V, K219Q }\end{array}$ & K101E, G190S \\
\hline 14. & Mn-35-A;HF679253 & $b$ & 2005 & $2005 / 2011$ & $A$ & 2012 & - & A62V, M184V & K103N \\
\hline
\end{tabular}




\begin{tabular}{|c|c|c|c|c|c|c|c|c|c|}
\hline 15. & MnObl-54A1 & g & 2011 & $2011 / 2011$ & A & 2012 & - & M184V & K101EQ, G190A \\
\hline 16. & $\begin{array}{l}\text { MnObl-67; } \\
\text { HF679213 }\end{array}$ & g & 2004 & $2004 / 2011$ & URF & 2012 & - & $\begin{array}{l}\text { M41L, D67N, } \\
\text { T69N, K70R, } \\
\text { M184V, T215F, } \\
\text { K219E }\end{array}$ & K101H, G190S \\
\hline 17. & $\begin{array}{l}\text { Gom-23-A; } \\
\text { HE657434 }\end{array}$ & g & 1996 & 2005/2010 & A & 2011 & - & $\begin{array}{l}\text { M41L, E44D, } \\
\text { V75M, M184V, } \\
\text { L210W, T215Y }\end{array}$ & $\begin{array}{l}\text { A98G, K101E, } \\
\text { Y181C, G190S }\end{array}$ \\
\hline 18. & MnObI-92A1; & g & 2004 & $2004 / 2009$ & A & 2012 & - & M184V, T215F & K101E, G190S \\
\hline
\end{tabular}

Table 2: High/level resistance mutation at patients on HAART (children).

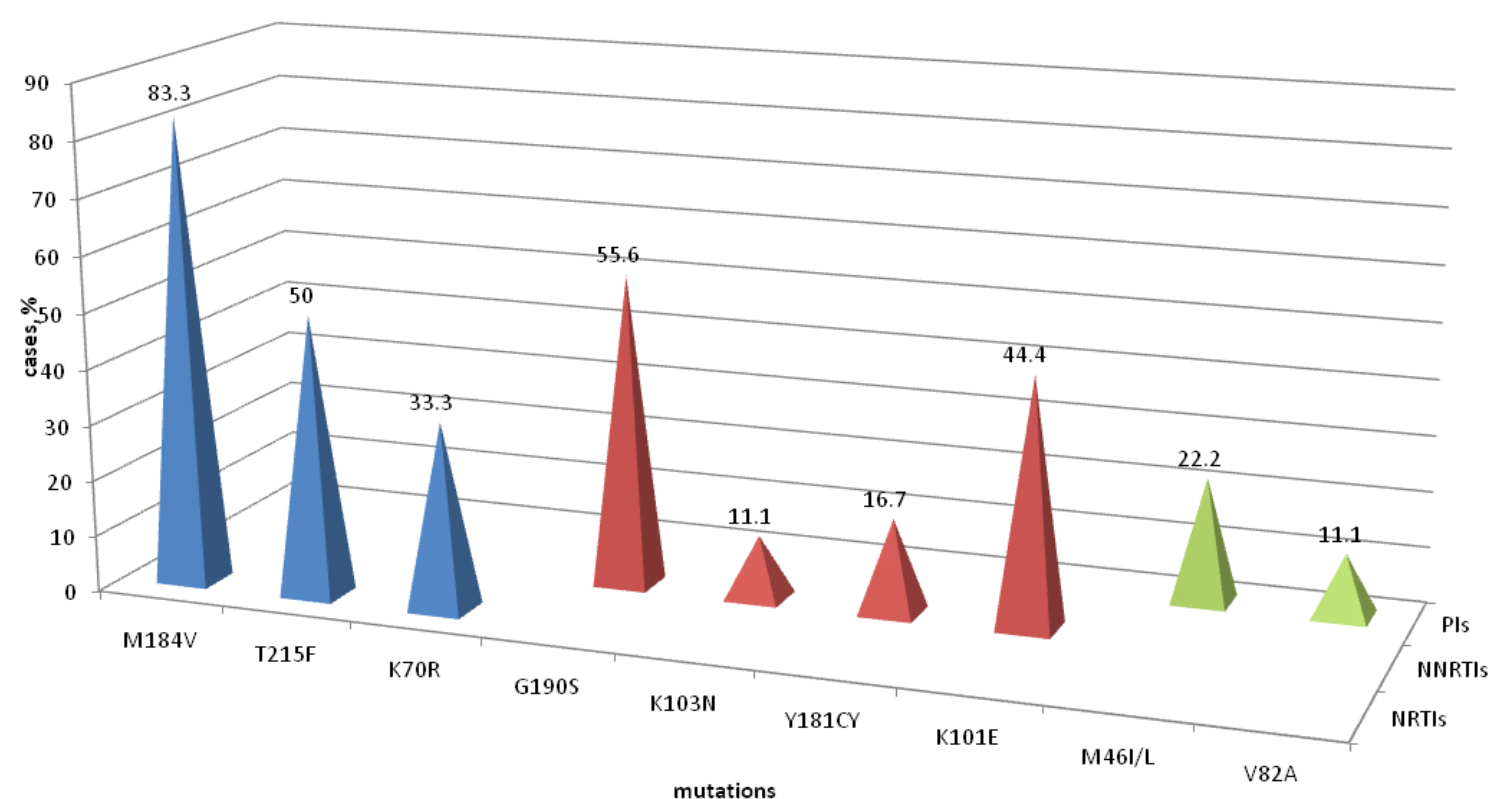

Figure 3: Resistance mutations identified in patients on HAART (children)

region and 3 patients from the Vitebsk region.

55 (93.2\%) patients were identified as carriers of human subtype A HIV-1, 3 (6\%) were infected with recombinant forms of CRF06_cpx and CRF03_AB and one patient (2\%) was infected with subtype B (Table 1).

9 (49.2\%) patients have been living with HIV/AIDS for more than 10 years, $25(42.4 \%)$ patients were infected between 5 and 9 years and $5(8.4 \%)$ patients carried the disease for less than 5 years. 40 (25 male and 15 female) during the period of the study received AZT +3TC+NVP (EFV), 7 (5 male and two female) were on the scheme FTC+TDF+NVP, 12 patients were on other schemes and one patient did not get the drugs.

The analysis of the pol gene sequencing results showed that most of the patients in the test group developed resistance mutations to nucleoside (NRTIs) and non-nucleoside (NNRTI) reverse transcriptase inhibitors (Figure 2).

In 38 (64.4\%\%) patients (24 male and 14 female) mutation M184V/I was identified, which causes high level HIV resistance to NRTIs. In 27 (45.8\%) patients ( 13 female and 14 male) K103N mutation was detected, which determines high level HIV resistance to NNRTIs. In 20 (33.9\%) patients (16 male and 4 female) we found G190G/S/A mutation, which is associated with the emergence of high-level resistance mutations to NNRTI.
$11(18.6 \%)$ and $8(13.6 \%)$ patients were identified to have $\mathrm{T} 215 \mathrm{~F}$ and K70R mutations, which determine reduction in susceptibility to AZT, d4T and ADC, as well as AZT, d4T and TDF, respectively. In 15 (25.4\%) patients ( 3 female and 12 male) we detected a combination of mutations $\mathrm{M} 184 \mathrm{~V}+\mathrm{G} 190 \mathrm{~S}$.

In $11(18.6 \%)$ HIV infected patients (6 female and 5 male) was detected combination of resistance mutations K103N+M184V.

More often, in $49.2 \%$ of cases (29 people), high-level resistance mutations were detected in patients who have the disease experience of 10 or more years. $25(42.3 \%)$ patients had experience of the disease between $5-9$ years. And only $5(8.5 \%)$ patients were infected less than 5 years ago.

In $76.3 \%$ cases ( 45 people) - resistance mutations were detected in patients who have experience of 2-4 years of therapy. 10 patients had a length of less than 2 years of treatment and 4 patients were treated with drugs for more than 5 years.

Of the 18 children-patients, born to HIV-infected mothers, 9 were boys aged $10.2 \pm 1.7$ and 9 girls (Table 2). 7 children were from the Minsk region, 5 from the Gomel region, 2 children were from the Mogilev region, 2 from the city of Minsk, 1 child from the Vitebsk region and 1 child from the Brest region. 16 children were infected with HIV-1 subtype A1, one - CRF02_AG and one child - URF (Table 2). In 15 $(83.3 \%)$ cases ( 9 girls and 6 boys), we detected HIV resistance mutation 
Citation: Eremin V, Gasich E, Sasinovich S, Suetnov O Kucherov I (2013) HIV Drug Resistance at Patients on HAART and Transmitted HIV Drug Resistance (tHIVDR) in Treatment Naive Patients in Belarus. J AIDS Clin Res 4: 258. doi: 10.4172/2155-6113.1000258

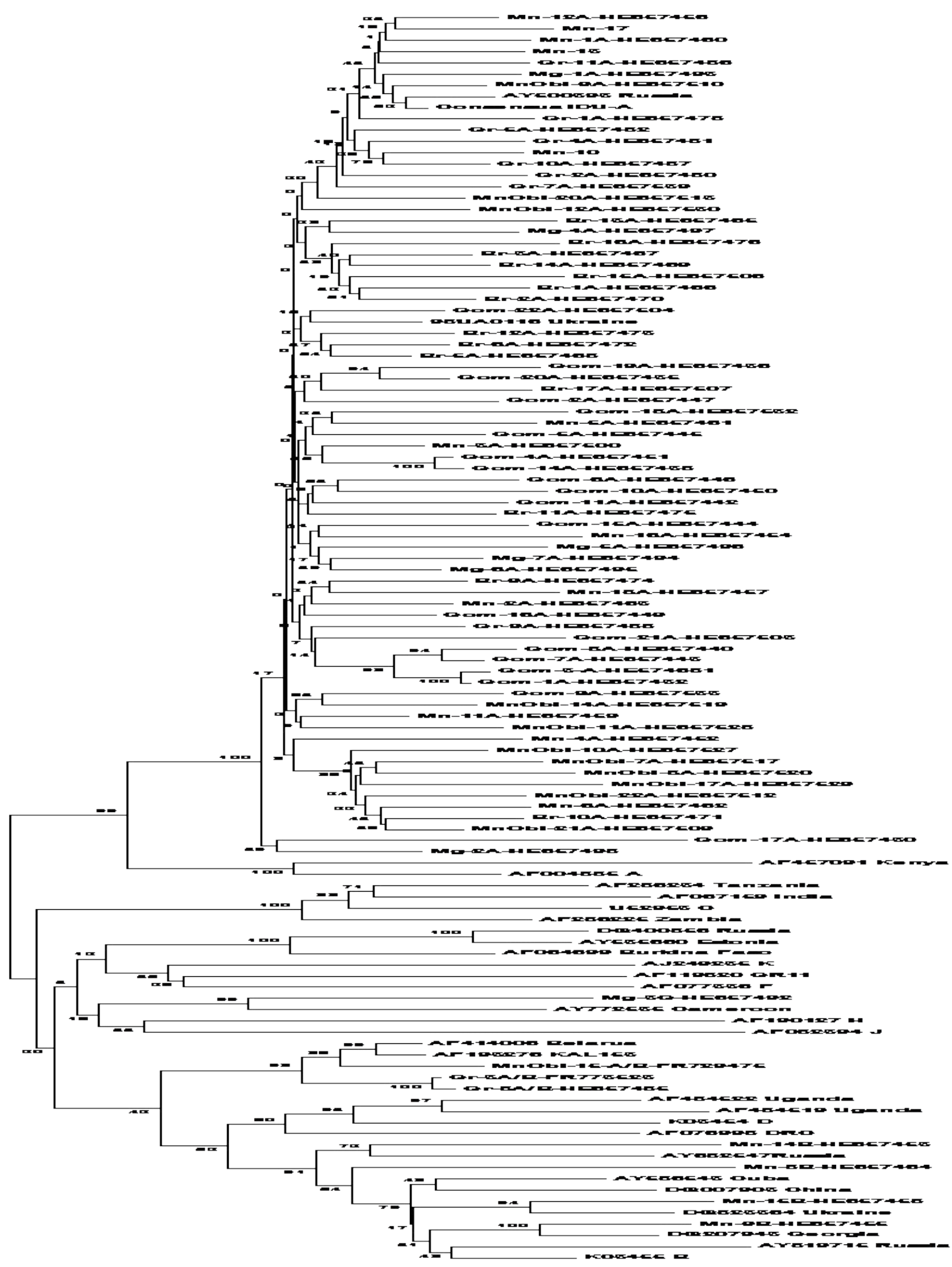

Figure 4: Phylogenetic analysis of HIV-1 gene pol sequences from a newly diagnosed HIV-infected antiretroviral naïve patients isolates. Reference sequences of HIV-1 and viruses specific for the epidemic in the former Soviet Union and Belarus as well as A-K subtypes are included. Reference sequences of HIV-1 subtypes are labeled by their GenBank accession numbers. For IDU-A viruses, the consensus of viruses from Svetlogorsk1 is used as a reference. The neighbor-joining tree were built by using the Kimura two-parameter distance estimation method and pairwise gap deletion. Bootstrap analysis was done with 100 replications, values above 70 are shown 


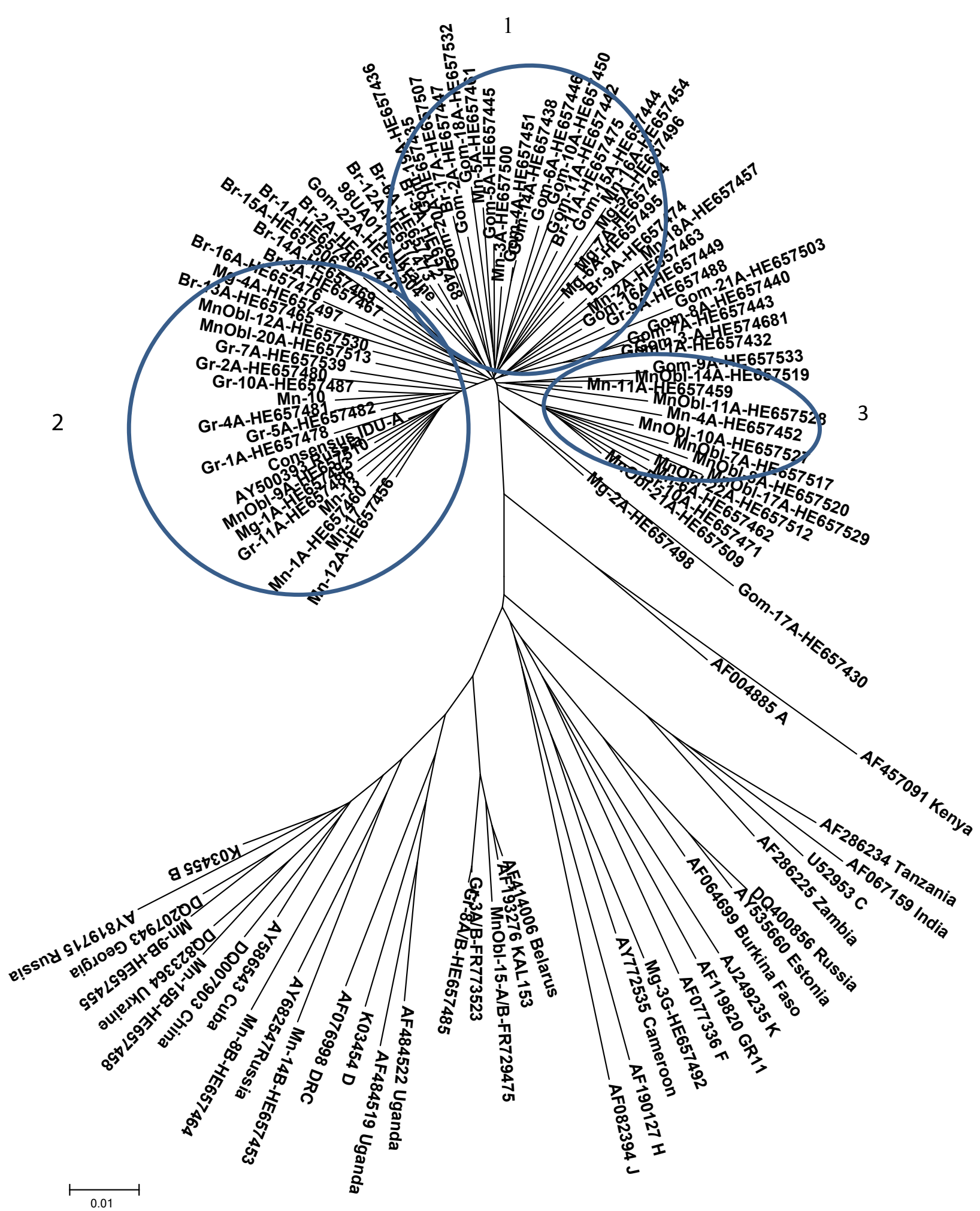

Figure 5: Phylogenetic analysis of HIV-1 gene pol sequences from a newly diagnosed HIV-infected antiretroviral naïve patients isolates. Reference sequences of HIV-1 and viruses specific for the epidemic in the former Soviet Union and Belarus as well as A-K subtypes are included. Reference sequences of HIV-1 subtypes are labeled by their GenBank accession numbers. For IDU-A viruses, the consensus of viruses from Svetlogorsk 1 is used as a reference. The neighbor-joining tree were built by using the Kimura two-parameter distance estimation method and pairwise gap deletion. Bootstrap analysis was done with 100 replications, values above 70 are shown. 
$\mathrm{M} 184 \mathrm{~V}$, which determines, as it was mentioned above, a high level of resistance to NRTIs. In $10(55.6 \%)$ and $2(11.1 \%)$ cases mutations in position G190S and K103S were found, respectively defining, a high level of resistance to NNRTIs (Figure 3). In two children mutation Y188L was identified, which leads to the emergence of high-level HIV resistance to NNRTI. In 4 cases resistance mutation at position M46I/L was identified, which, in presence of other mutations, leads to a reduced sensitivity to HIV protease inhibitors: indinavir (IDV/r), nelfinavir (NFV), fosamprenavir (FPV/r), lopinavir (LPV/r) and atazanavir $(\mathrm{ATV} / \mathrm{r})$.

In $9(50 \%)$ and $6(33.3 \%)$ cases mutations T215F and K70R were detected. In 8 and 3 cases mutations K101EQH and Y181CFGV were detected, respectively. K101EQH mutation reduces of HIV-1 sensitivity to NVP, EFV and ETR, and Y181C/I/V mutation leads to a high level of resistance to NVP and DLV.

In 14 children ( 8 girls and 6 boys) various combinations of mutations leading to a high resistance were found (Table 2).

Most often, high-level resistance mutation were identified in children with the disease experience more than 10 years $(9 / 50 \%)$ and 5-9 years $(8 / 44,4 \%) .8$ children were detected to develop highlevel resistance mutations after 2-4 years from the start of treatment, 6 children showed mutations developing after less than 2 years of therapy.

Thus, as it was shown by the undertaken research and studies adults and children receiving HAART are detected to develop M184V and G190S mutations approximately with the same frequency. At the same time, among children mutation $\mathrm{K} 103 \mathrm{~N}$ is less often. As research shows, the emergence of resistance mutations is associated with the length of the period of experience of the disease. More than $90 \%$ of all patients, $94.4 \%$ children and $91.5 \%$ adults, who were detected with a high-level resistance mutations, had long period of registered disease experience - more than 5 years (the length of the disease period is considered to start from the day the patient is officially diagnosed to be infected with HIV/AIDS and his/her registration in Western blot, but, obviously, patients can be identified and registered already having an advanced HIV infection, cases of pre-AIDS stage also must be taken into account).

Thus, timely, early-stage identification of HIV/AIDS infection and prompt prescription and provision of the patient with most efficient antiretroviral drug therapy schemes will substantially improve quality of our help to this category of patients. Most frequently mutations were detected in patients after 2-4 years of treatment.For example, in $45(76.3 \%)$ adult patients and 8 (50\%) children high-level resistance mutations were detected after 2-4 years of taking ART. We are seriously worried by the fact that in 6 children (33.3\%) high-level resistance mutations were identified in less than two years from the start of the therapy. This fact highlights the necessity of strong and careful monitoring of children receiving ART, who live in problem families.

In general, we can talk about a good patient adherence to therapy, only $18.8 \%$ of the patients (77 of 313 ) had resistance mutations of high risk. 39 (66.1\%) of 59 adult patients are classified as IDUs, who, according to general experience, do not always express high degree of adherence to therapy [3-5].

96 blood samples were collected from newly diagnosed HIVinfected antiretroviral naïve patients genotype testing was successfully performed on 82 samples. Of 82 samples 43 (51.8\%) were from females. Median age - $32-36$ years; $81.7 \%$ (67) of people were diagnosed as HIV infected 1-3 years ago. Of total of 82 pol sequences analyzed 74 (90\%) belong to HIV-1 subtype A; four (4.9\%) - subtype B; three (3.7\%) to CRF03_AB; and one was subtype G (Figure 4). The viruses carried to subtype A, formed 3 big groups (Figure 5). The Greatest first group including 35 samples, consisted basically of the specimens received from a Svetlogorsk city of the Gomel oblast where, as it is known, in 1996 there was HIV-infection outbreak among IDUs and circulates, a so-called "Svetlogorsk" a HIV-1 subtype A1 variant [6]. Average p-distances in group were 0.039 and fluctuated within 0.012-0.069 that specifies as in circulation before they brought virus, and new cases of an infection. The second group consisting of 24 samples formed patients from the Grodno oblast and a city of Minsk, mainly. There was an outbreak of a HIV-infection among IDUs in the Grodno oblast in the late nineties. Samples were formed around AF413987 (Ukraine), AY500393 (Russia) and consensus-IDU-A. Average p-distances were 0.040 and fluctuated from 0.026 to 0.056 that specifies in virus long circulation in the given population of patients and separate new cases of an HIV-infection. The third group including 12 samples formed patients from the Minsk oblast, basically from the cities of Soligorsk and Slutsk where there was HIV-infection outbreak among IDUs in the beginning of 2000th. Average p-distances between samples in group were 0.039 and fluctuated from 0.023 to 0.056 . The HIV- 1 from the given group has been designated as «Soligorsk» isolate. Samples AF873832 and FR87227, FR873141 have lain separately from other groups that specifies in independent penetration of a virus on territory of the country.

Apart from resistance mutations included in WHO 2009 list of SDRM, 6 samples (7.3\%) had other resistance mutations which can be classified as 'minor' or 'other' according to HIVDR database of Stanford University: L10V - PI minor mutation associated with resistance to most PIs when present with other mutations; L33F - PI minor mutation selected by FPV/r, DRV/r, LPV/r, ATV/r, and TPV/r, and contributes decreased susceptibility to these PIs; V118I - accessory mutation usually occurred with multiple TAMs and contributes some resistance to each of the NRTIs including 3TC and FTC; T74S is 'other' mutation associated with reduced NFV susceptibility; and V108I accessory mutation, causes low-level resistance to NVP and EFV.

According to the WHO recommendations in case of low prevalence of tHIVDR investigation should be repeated after 2 years, no change of ART regimens is required. However ongoing monitoring of ART programme focusing on risk factors such as continuous access to services, drug quality and supply, prescribing practices, adherence, adverse effects, and treatment failures is absolutely necessary.

\section{Acknowledgments}

This work was funded by the Global Fund, international technical assistance project "Prevention and Treatment of HIVIAIDS in the Republic of Belarus and Belarusian Office of World Health Organization."

\section{References}

1. Boyer PL, Sarafianos SG, Amold E, Hughes SH (2002) Nucleoside analog resistance caused by insertions in the fingers of human immunodeficiency virus type 1 reverse transcriptase involves ATP-mediated excision. J Virol 76: 9143-9151.

2. Casado JL, Moreno A, Hertogs K, Dronda F, Moreno S (2002) Extent and importance of cross-resistance to efavirenz after nevirapine failure. AIDS Res Hum Retroviruses 18: 771-775.

3. Kellam P, Larder BA (1995) Retroviral recombination can lead to linkage of reverse transcriptase mutations that confer increased zidovudine resistance. J Virol 69: 669-674.

4. Ishizaki A, Cuong NH, Thuc PV, Trung NV, Saijoh K, et al. (2009) Profile of HIV type 1 infection and genotypic resistance mutations to antiretroviral drugs in 
Citation: Eremin V, Gasich E, Sasinovich S, Suetnov O Kucherov I (2013) HIV Drug Resistance at Patients on HAART and Transmitted HIV Drug Resistance (tHIVDR) in Treatment Naive Patients in Belarus. J AIDS Clin Res 4: 258. doi: 10.4172/2155-6113.1000258

Page 9 of 9

treatment-naive HIV type 1-infected individuals in Hai Phong, Viet Nam. AIDS Res Hum Retroviruses 25: 175-182.

5. Alexander CS, Dong W, Schechter MT, O'Shaughnessy MV, Strathdee SA, et al. (1999) Prevalence of primary HIV drug resistance among seroconverters during an explosive outbreak of HIV infection among injecting drug users. AIDS 13: 981-985.

6. Lukashov VV, Karamov EV, Eremin VF, Titov LP, Goudsmit J (1998) Extreme founder effect in an HIV type 1 subtype A epidemic among drug users in Svetlogorsk, Belarus. AIDS Res Hum Retroviruses 14: 1299-1303. 